\title{
Re-Implantation of Total Hip Replacement (THR) with Burch-Schneider (BS) plate in 2003-2007 - Midterm Results at Our Institution
}

\author{
S Magersky* \\ Department of Orthopaedics, $1^{\text {st }}$ Faculty of Medicine, Charles University and Na Bulovce Hospital, Czech Republic
}

*Corresponding author: S Magersky, Department of Orthopaedics, $1^{\text {st }}$ Faculty of Medicine, Charles University and Na Bulovce Hospital, Budínova 2, Praha 8, 180 81, Czech Republic

\section{ARTICLE INFO}

Received: 崖 March 01, 2019

Published: 慧 March 12, 2019

Citation: S Magersky. Re-Implantation of Total Hip Replacement (THR) with Burch-Schneider (BS) plate in 20032007 - Midterm Results at Our Institution. Biomed J Sci \& Tech Res 15(5)2019. BJSTR. MS.ID.002766.

Keywords: Pelvis; Hip \& Femur; Joint Replacement - Secondary

Abbreviations: BS: Burch-Schneider; AP: Anteroposterior; THRs: Total Hip Replacements

\section{ABSTRACT}

Here, we report the midterm results of re-implantation using Burch-Schneider (BS) plate for Paprosky bone defects IIIa and IIIb. The optimal treatment of pelvis discontinuity remains controversial.

Materials and Methods: Five hundred and forty-nine total hip re-replacement surgeries were performed at our institution, the Orthopaedic Clinic of the Bulovka Hospital, between 2003 - 2007. Follow-up ranged from 5 to 10 years. 243 of the surgeries involved acetabular cup re-implantations, with 77 (31.6\%) of those having used the BS cage. In all cases, the acetabular bone loss was assessed according to Paprosky classification. Preoperative procedures included anteroposterior pelvis x-ray pelvis, with determination of the proximalization of the center of rotation, osteolysis of the os ischii and teardrop, and position of the implant in relation to Köhler's line. During the operations, the remaining bone stock and pelvis discontinuity was defined.

Results: Early aseptic loosening as a sequel of insufficient proximal screw fixation due to bone loss occurred in 7\% (defined as asymptomatic loosening); among these cases, $4.2 \%$ had complete loosening necessitating revision surgery and $2.8 \%$ had an infection complication necessitating extraction of the BS cage. The affected patients were $72 \%$ females, with median age of 75 years. Kaplan-Meier analysis showed $50 \%$ survival > 9 years, with survival out to 16 years (maximal monitoring time of the study) being $36 \%$.

Conclusion: For good long-term results of BS plate implantation it is mandatory to use bone allografts with optimal implant stability so as to achieve good bone osteointegration.

\section{Introduction}

The Burch-Schneider (BS) plate (Figure 1) has been in constant use since its introduction in 1974. In 1987, however, the material was switched from steel to titanium, with the aim of improving its utility and long-term outcome. Our institution commonly uses the BS plate for pelvis reconstructions. In the preoperative planning stage, we routinely perform anteroposterior (AP) x-ray of the pelvis to determine the pelvic defects, proximalization of the center of rotation, osteolysis of the os ischii and teardrop, and the position of implants with respect to the Köhlers ilioischial line. All of these parameters can influence re-implantation of the acetabular component, particularly as they help to determine the underlying acetabular defects. We also routinely use the Paprosky classification system [1] when assessing which spherical acetabular components to use for re-implantation (Figure 2), considering every degree for its effect on the stability of the spherical component to be implanted. Most of our cases were IIIa than IIIb as defect of the acetabulum. For patients with Paprosky defect IIIa-defined as a proximolateral defect without pelvic discontinuity - the plate is better implanted into pelvis, as it will overcome the loss integrity in the posterior wall due to the defect. Considering the literature, 
Regis et al. [2] reported the protrusion and deficiency of the medial wall acetabulum and that the defect can effect more than $50 \%$ of resting bone. Scott et al. [3] also reported that excessive defects of the acetabulum, without healing potential, are indicators for use of bone grafts in the reconstruction procedure. On the other hand, Hur et al. [4] reported on a mechanical aspect, in that osteosystesis of the pelvis occurs due to pelvic discontinuity with press-fit acetabulum when the BS cage is used, even when the BS cage did not present any superficial results (i.e. functional outcomes).

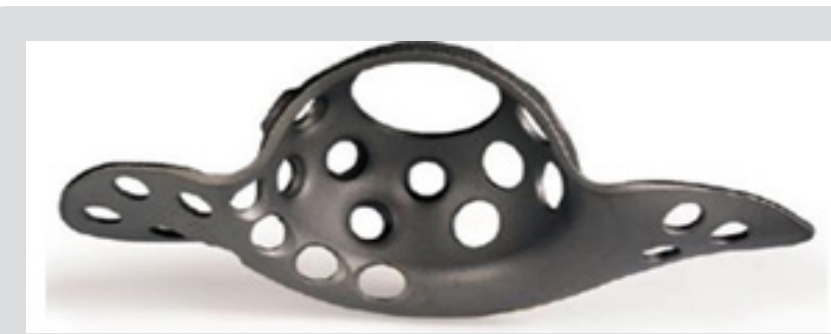

Figure 1: BS plate.

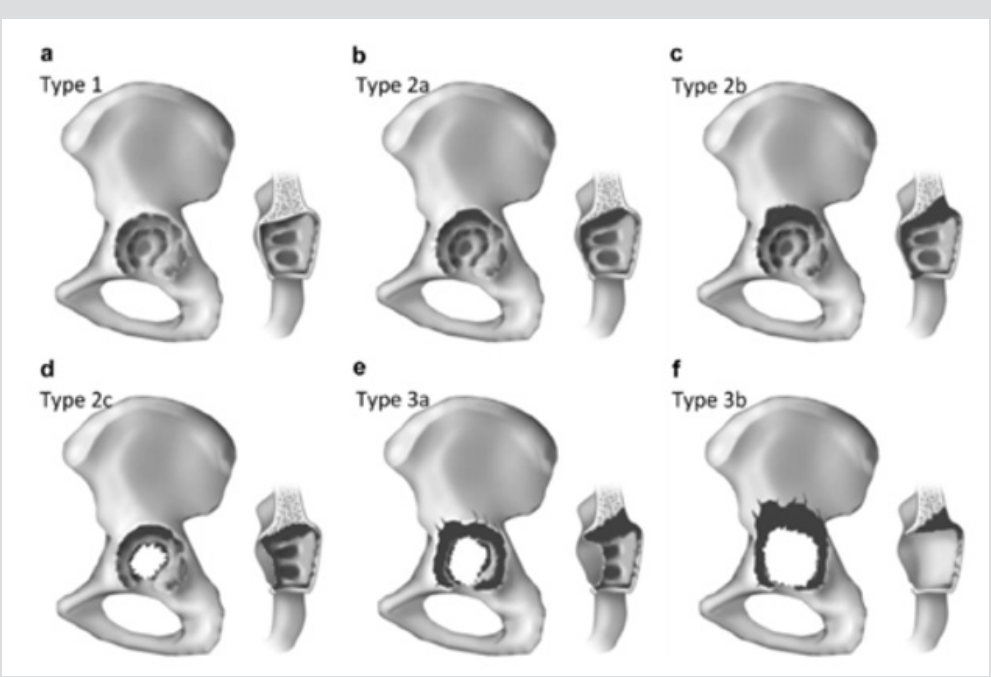

Figure 2: Paprosky classification of acetabular defects.

Finally, Peters et al. [5] had earlier noted poor long-term results for the BS plate in resolving pelvic discontinuity. The stability of the BS plate can be assessed by x-ray, and the imaging findings can help to predict its duration over the long-term. To this end, Gill et al. [6] showed plate instability as being indicated on x-ray by fracture of the proximal screws in the plate, together with proximal-migration of more than $5 \mathrm{~mm}$, and with progression of radiolucent lines. Moreover, Van den Linde and Tonino [7] showed that fracture of the proximal screws without subsequent migration of the plate or change of inclination on x-ray does not equate to plate loosening. Our x-ray analyses agreed with these reported parameters (Figure 3). In addition, Gross et al. [8] showed that resorption of the bone grafts implanted into the pelvis upon re-implantation served as a marker of plate stability, as evidenced by x-ray imaging. This study was designed to Gross et al. [8].

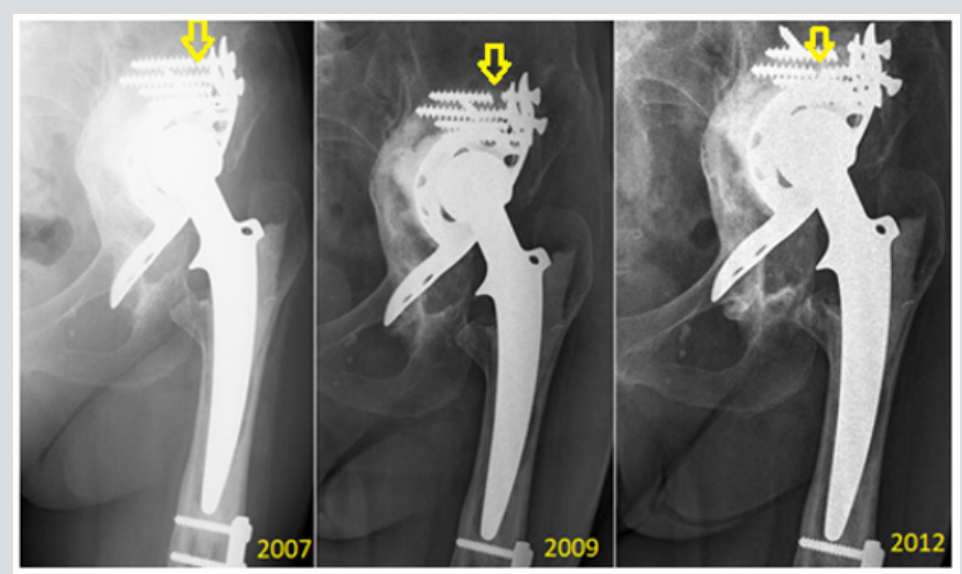

Figure 3: Representative case of fracture located proximally in the BS plate but without loosening. The three x-ray images were taken over a 6-year period, as indicated. The fractured screws are indicated by yellow arrows. 


\section{Materials and Methods}

The operative techniques for implantation of a BS plate are designed according to the presence of pelvic disjunction, i.e. Paprosky IIIa or IIIb classifications. In the case of pelvic disjunction, there can be screw fixation in the distal part of the pelvic plate and into the osischii. Although this bridging plate technique is described throughout the literature, we use it seldomly, and only in patients with low load demand lifestyle because of the potential to loosen earlier when impaction of the osteointegration into the pelvis is inadequate. The trabecular-titan system is an alternative technique but is associated with substantially greater monetary cost. A third technique involves the impaction to os ischii, analogous to revision of the spherical acetabula. What we advocate is impaction into the os ischii and a 3-point fixation of the montage. In all cases, it is necessary to use bone grafts, particularly around the plate area and to fill in any bony defects; in this manner, excessive stability is achieved for the plate implant inside pelvis.

\section{Results}

In our institution, 549 hips were re-implanted in the 4-year period from 2003 to 2007, with 243 of those re-implantations involving an acetabular component. BS plate was used in 78 (28.8\%) of those latter cases, with only 8 of the patients being lost to follow-up. Also, in our institution, since 2000, we have preferred to perform non-cemented total hip replacements (THRs) in younger patients (under 70 years-old), and we have noted the need to perform re-implantation for subsequent defects, which tend to be larger, and especially so with the acetabular component (Figure 4). All patients with BS plate re-implantation were assessed by x-ray of the pelvis to identify cases of asymptomatic loosening; only 5 cases (7\%) were found. In follow-up, the patients were re-operated (two times in total for $4.2 \%$ of the cases to address complete loosening or for an infectious complication in 2 cases (2.8\%), with one needing full extraction of the BS plate. (Figure 5) provides an excellent representation of a case of the BS plate loosening itself.
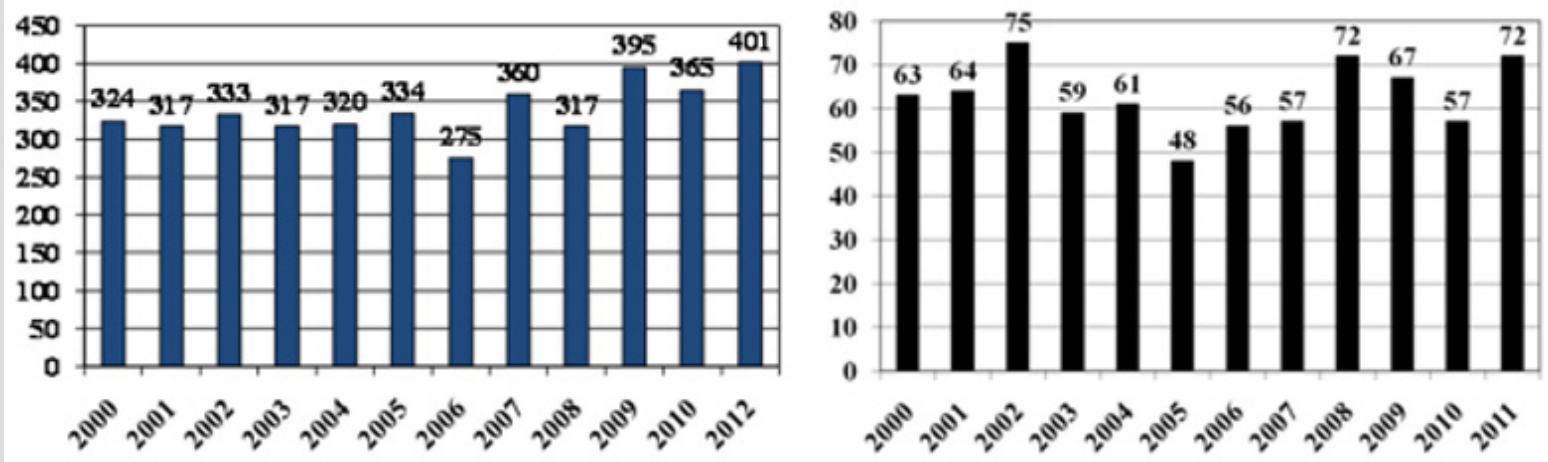

Figure 4: Increasing trend towards THR and re-THR over the past decade at our institution

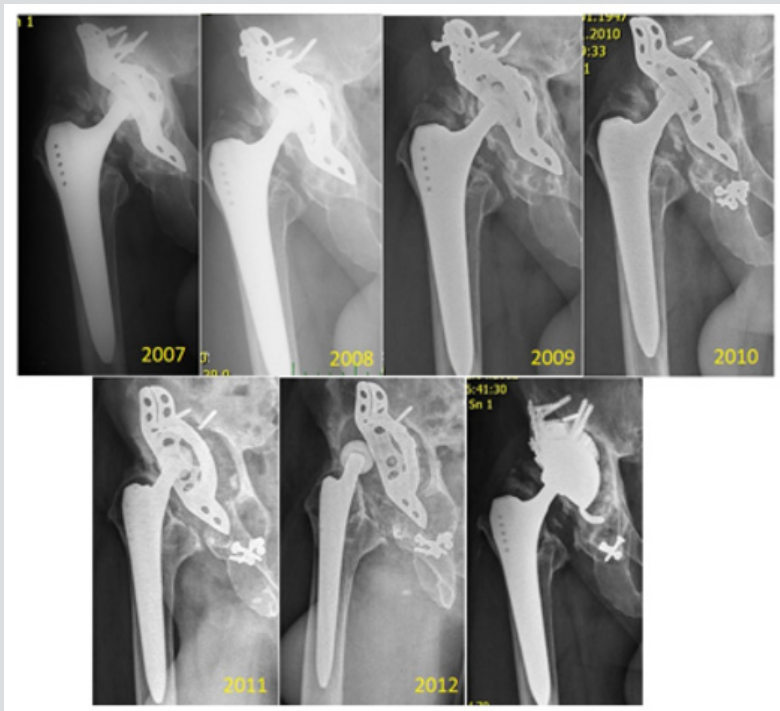

Figure 5: Representative case of mechanical loosening of the BS plate. The proximal screws were fixed into defective bone, and a final revision was made by implantation of a hemispheric acetabular modulus. The final panel shows the results of the re-reimplantation (occurred and imaged in 2012). 
The migration occurred proximally, with breakage of the proximal screws and migration of the plate (from 2009). Then, a mixture of the screws is seen in the bottom of the joint space (2010) and luxation (2012). In the same year (2012), we re-admitted the patient for re-reimplantation and implanted a hemispheric modulus with excenter and larger revision stem. In Figure 6, a case of mechanical loosening of the BS plate is presented. This event does not, generally, lead to positional plate loosening. Instead, the plate protrudes into the pelvis slightly, though it is still capable of full-weight bearing.

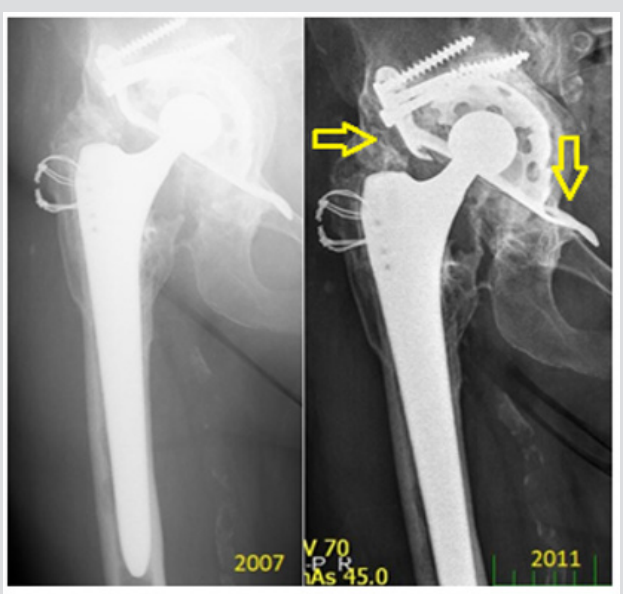

Figure 6: Representative case of mechanical loosening of the BS plate involving the proximal region. No BS plate failure occurred in such a case. Yellow arrows denote the stability of the implant at 4-year post-implantation.

\section{Bone Cement}

The use of bone cement is not supported in the literature. It is typically associated with early failure of the implant and no osteointegration. Our case series included several experiences of aseptic loosening. For this analysis we focused on early loosening events, which occurred as a result of insufficient fixation by screws due to bone defects or osteoporosis. The use of bone cement as a filament of bone defects, instead of bone allografts, did not produce very good results, even by firm osteosynthesis. Moreover, this technique left patients with functional ability that could only handle low load demand (Figure 7). In addition, in cases with successful allograft and achievement of good primary stability of the plate, no failure occurred when there was fracture of the plate. Primary mechanical stability of the BS plate is essential for integration of the bone allografts into defects. For pelvic disjunction, use of the BS plate as a bridging plate remains controversial because of the overall lack of patients in our series and of long-term results for those who did receive it (Figure 8).

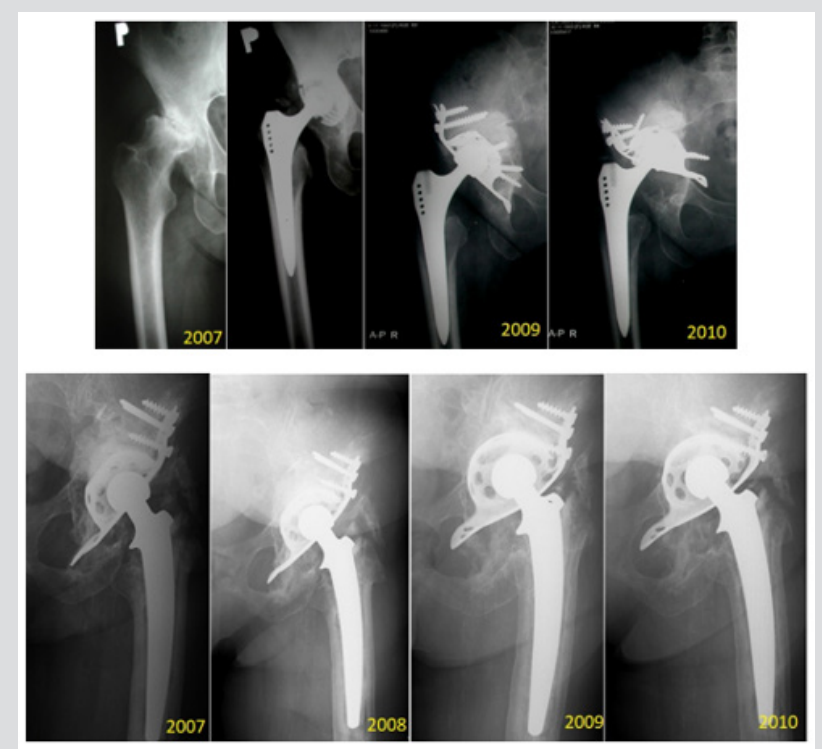

Figure 7: Representative case of implantation of the BS plate with cement filament instead of bone allograft leading to failure within 3 years. 


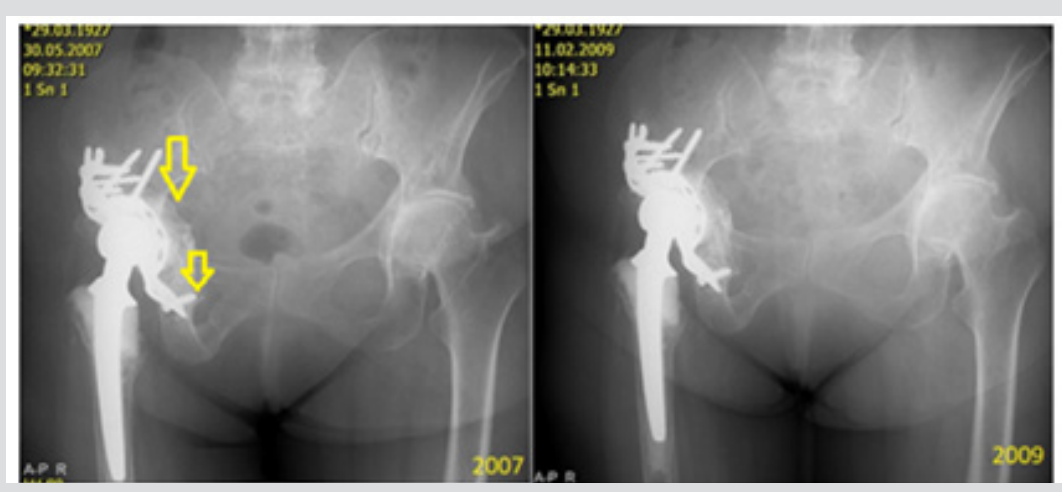

Figure 8: Representative case of pelvic discontinuity with use of BS plate as a bridging plate. The fixation involved the os ischii and good osteointegration is shown (arrows).

We had performed operations only from the lateral pelvic approach, and revision with pelvic approach was not necessary; this approach is even recommended for revision of pelvic vessels $[9,10]$. We performed preoperative angiography in cases with suspected vessel damage and subsequent likelihood of difficulty in operation; as such, this procedure was not performed routinely. We preferred to perform a preoperative computed tomography scan with filtration of the steel scatter. This technique helped us to assess the key feature of good, long-lasting duration, that being good integration of allografts, deep into the acetabulum. Good position and firm osteointegration of the plate are also essential, as they will promote creation of the bony layer inside the pelvis (Figure 9). Additional results are presented in Figures 9-14 and Tables 1-3.

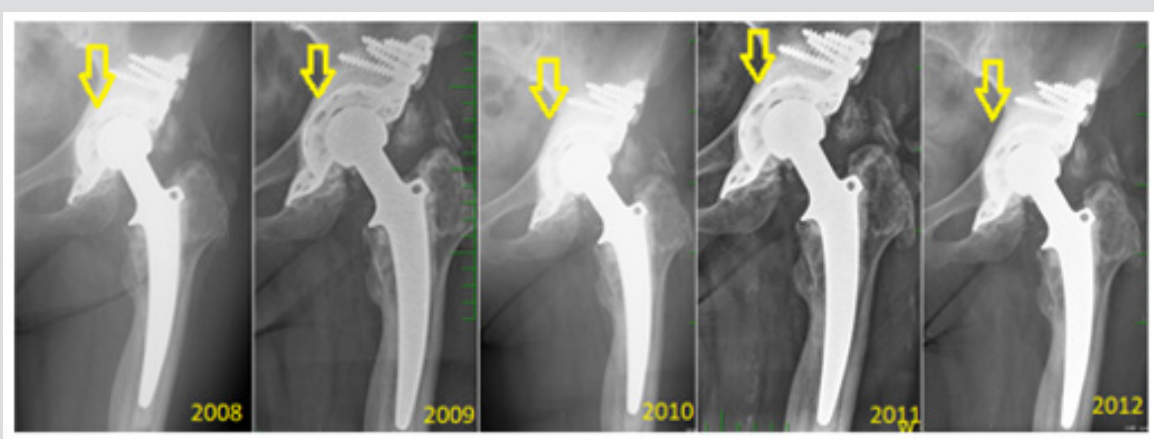

Figure 9: Representative case of good positioning of the BS plate and regular resorption of bony allografts. The key features are highlighted by arrows.

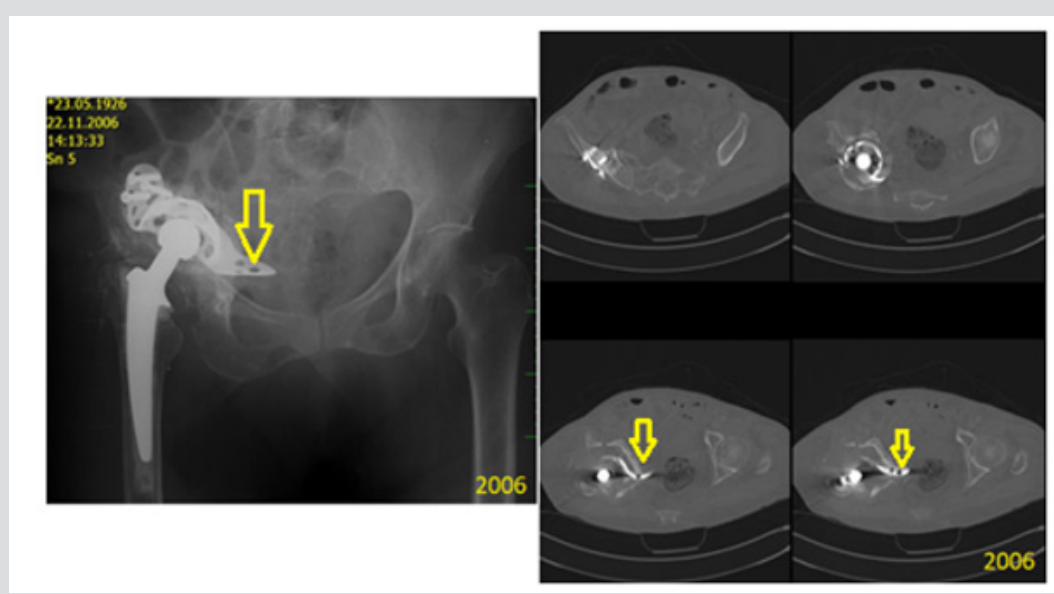

Figure 10: Representative case of failure of the BS plate. A, C: X-ray images. B, D: Computed tomography scan showing migration of the distal part of the plate into the pelvis (arrows). 


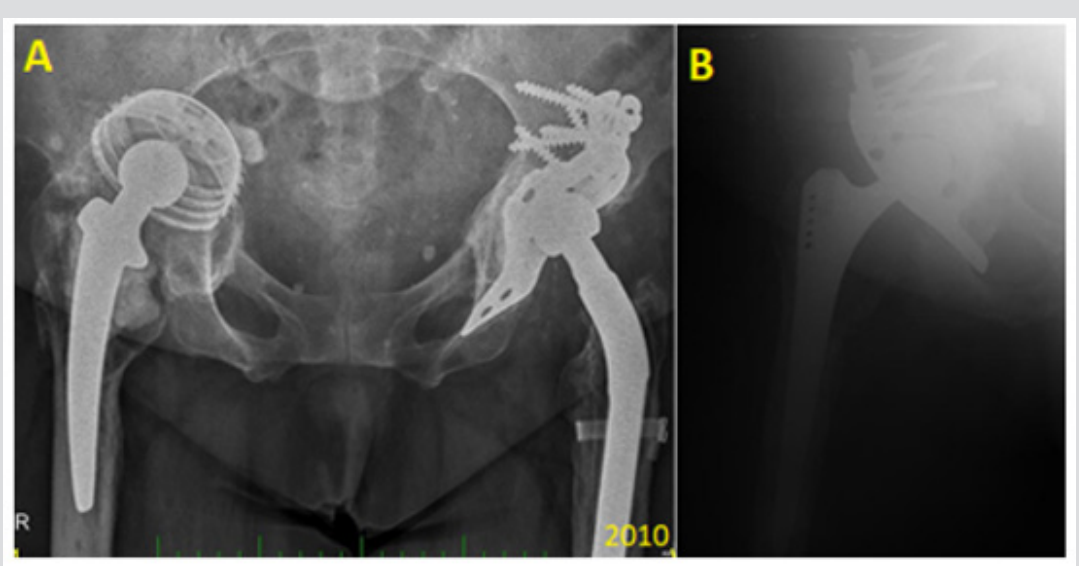

Figure 11: Representative case of a patient with double implantation, who experienced failure of a threaded cup (arrows) in right THR (A) and replacement of the BS plate (B). In panel A, protrusion of the acetabulum into the pelvis (threaded cup) is shown, which led to the replacement by the BS plate (shown in B).

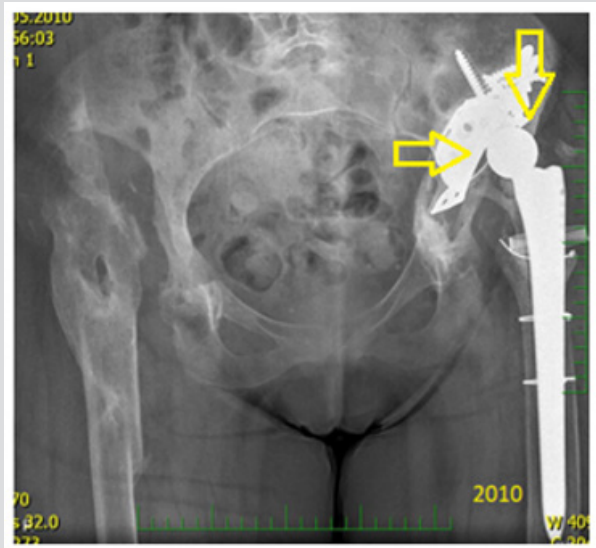

Figure 12: Representative case of failure of the BS plate. Protrusion into the pelvis with failure of the pelvic vessels is shown. The BS plate was in the subluxation position (yellow arrows).

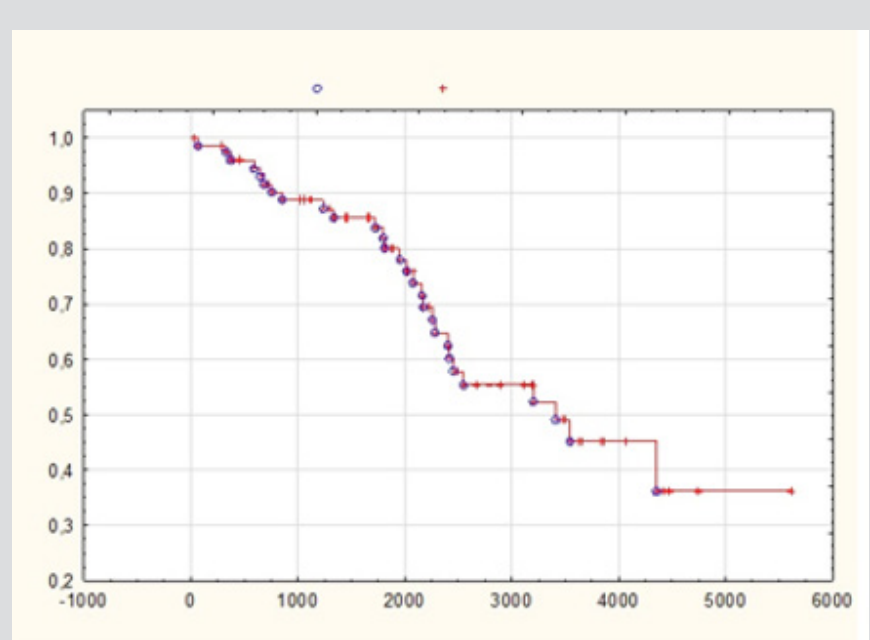

Figure 13: Kaplan-Meier survival analysis for the BS plate. After 9 years, the plate remained functional and in-place (i.e. survived) in half of the cases. Among those cases, $36 \%$ maintained surviving BS plate at 16 years (the maximum time of followup in our study). Y-axis, days; X-axis, cumulative Kaplan Meier curve. Blue circles, patients who completed follow-up; Red crosses, 'censored' patients (patients who died in follow-up). 


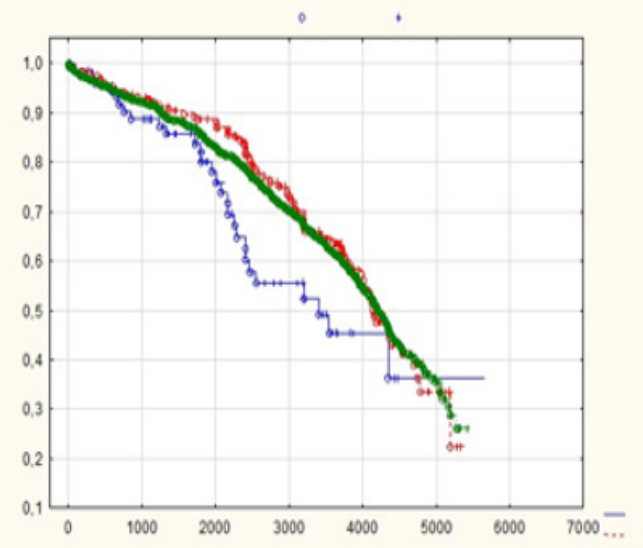

Figure 14: Cumulative survival of all implants. Similar survival rates were found for implants after re-implantation and implants without re-implantation. The rate for BS plate had a steeper downward slope, indicating its worse survival rate. Y-axis, days; X-axis, cumulative Kaplan-Meier curve. Blue circles, patients who completed follow-up; Red crosses, 'censored' patients (patients who died in follow-up). Blue solid line, BS plates; Red dashed line, re-implantation total; Green dotted line, THR without re-implantation.

Table 1: Death chart for patients with the BS plate. The maximum average of death after 6 years is shown, and it does not have to correlate with the re-implantation.

\begin{tabular}{|c|c|c|c|}
\hline Beginning of the interval, in days & Patients at-risk, $\mathbf{n}$ & Excluded patients, $\mathbf{D}$ & 2 \\
\hline 0 & 75 & 4 & 4 \\
\hline 365 & 71 & 2 & 2 \\
\hline 730 & 63 & 7 & 2 \\
\hline 1095 & 59 & 6 & 3 \\
\hline 1460 & 50 & 4 & 5 \\
\hline 1825 & 41 & 3 & 6 \\
\hline 2190 & 32 & 3 & 0 \\
\hline 2555 & 23 & 3 & 1 \\
\hline 2920 & 20 & 4 & 2 \\
\hline 3285 & 16 & 4 & 0 \\
\hline 3650 & 10 & 1 & 1 \\
\hline 4015 & 6 & 3 & 0 \\
\hline 4380 & 4 & 0 & 0 \\
\hline 4745 & 1 & 0 & 0 \\
\hline 5475 & 1 & 1 & \\
\hline
\end{tabular}

Table 2: Quantile supplementation for more precise interpretation of survival rates in days.

\begin{tabular}{|c|c|c|c|}
\hline Group & $\mathbf{7 5 \%}$ of patients surviving, in days & $\mathbf{5 0 \%}$ of patients surviving, in days & 25\% of patients surviving, in days \\
\hline BS plate & 2039 & 3347 & - \\
\hline overall re-implantation & 2874 & 4141 & 5091 \\
\hline all hips without re-implantation & 2627 & 4204 & - \\
\hline
\end{tabular}

Table 3: Quantile supplementation of the data, to provide more precise interpretation of survival rates in years. Regarding to the reliability of the curve in its right side - which lowers, the results are in median (50\%).

\begin{tabular}{|c|c|c|c|}
\hline Group & $\mathbf{7 5 \%}$ of patients surviving, in days & $\mathbf{5 0 \%}$ of patients surviving, in days & $\mathbf{2 5 \%}$ of patients surviving, in days \\
\hline BS plate & 5.6 & 9.2 & - \\
\hline overall re-implantation & 7.9 & 11.3 & 13.9 \\
\hline all hips without re-implantation & 7.2 & 11.5 & - \\
\hline
\end{tabular}




\section{Conclusion}

The algorithms of a decision to implant the BS plate are mainly derived from the preoperative $\mathrm{x}$-ray findings, on a patient-bypatient basis. It is important to determine whether there is a pelvic discontinuity. If we are to consider the pelvis as united, then it is important to consider perioperative findings of any acetabular deficit. Proximalization of the THR within $3 \mathrm{~cm}$ over the upper obturator line signifies that discontinuity is less probable. The perioperative step that proceeds this finding will then consist of a decision as to what is sufficient contact for the rest of affected bone with the implant. Finally, the Paprosky defect can be defined $[11,12]$. In patients with Paprosky defect IIIa, a trabecular titanium system may be used, which unfortunately is not suitable for use in individuals with low physical demand because of its cost. When we consider that stability is related to contact with the bone, then a Paprosky defect IIIb type becomes an important consideration.

The perioperative step will involve determining whether there is acute pelvic dissociation with sufficient bone for healing, or if there might be a chronic disjunction with insufficient bone to support good healing. Paprosky defect IIIa can be defined by a proximolateral migration of more than $3 \mathrm{~cm}$ over the obturator line, osteolysis of the os oschii (to $15 \mathrm{~mm}$ under the obturator line), destruction (even partially) of the teardrop, position of the component laterally from the Köhlers line, intact ilioischial and iliopubic lines, and lack of pelvic disjunction. Paprosky defect IIIb, on the other hand, can be defined by less than $40 \%$ contact between the bone and implant. Circumference bone defect involves more than half the circular portion of the acetabulum (between 9-5 hours), lysis of the os ischii of more than $15 \mathrm{~mm}$ under the upper obturator line, complete destruction of the teardrop, medial migration from the Köhlers line, and proximomedial migration of more than $3 \mathrm{~cm}$ from the obturator line.

Despite the low survival rate of the BS plate among our case series, we consider it to be of benefit for many patients, at lower costs than other implants. Our Kaplan-Meier survival analysis of the BS plate showed that after 9 years over half of the BS plates remained functional and in-place, with $36 \%$ of those remaining as such out to the maximum time of follow-up (16 years). The patient death analysis showed that the maximum deaths occurred up to the 6 th postoperative year, and these cases were not related to the implantation. Unfortunately, we do not have detailed records on the precise non-implantation death causes. The collective results from our case series are not very supportive of the use of BS plate, but we retain our belief in the potential of it as a solution for patients who do not have any other option for walking. To improve the potential for achieving longer survival of the implant it is necessary to use bone allografts and to ensure good primary stability of the plate. Our recommendation is to use, at minimal, 5-6 screws (observational data) firmly fixed into the proximal part of the plate. Our overall recommendation is to impact the lower part of the plate into the os ischii and to ensure the center of rotation.

\section{References}

1. Paprosky WG, Perona PG, Lawrence JM (1994) Acetabular defect classification and surgical reconstruction in revision arthroplasty: a 6-year follow-up evaluation. J Arthroplasty 9(1): 33-44.

2. Dario Regis, Bruno Magnan, Andrea Sandri, Pietro Bartollozi (2008) Long-Term Results of Anti-Protrusio Cage and Massive Allografts for the Management of Periprosthetic Acetabular Bone Loss. The Journal of Arthroplasty 23(6): 826-832.

3. Scott M Sporer, Michael O Rourke, Wayne G Paprosky (2005) The Treatment of Pelvic Discontinuity During Acetabular Revision. The Journal of Arthroplasty 20(4): 79-84.

4. Hur J, Coburn JC, Mott Bliss J (2005) Biomechanical comparison of acetabular construct in pelvis discontinuity. Presented at the Anual Meeting of the American Academy of Orthopaedic Surgerons, USA.

5. Peters CL, Curtain M, Samuelson KM (1995) Acetabular revision with the Burch-Schneider antiprotrusio cage and cancellous allograft bone. J Arthroplasty 10: 307.

6. Gill TJ, Sledge JB, Müller ME (2000) The management of severe bone loss using structural allograft and acetabular reinforcement devices. J Artroplasty 15(1): 1-7.

7. Van der Linde, Tonino A (2001) Acetabular revision with impacted grafting and a reinforcement ring. 42 patients followed for a mean of 10 years. Acta Orthop Scand 72(3): 221-227.

8. Gross AE (1999) Revision arthroplasty of the acetabulum with restoration of bone stock. Clin Orthop 369: 198-207.

9. Jonathan N Sembrano, Edward Y Cheng (2008) Acetabular Cage Survival and Analysis of Factors Related to Failure. Clin Orthop Relat Res 466(7): 1657-1665.

10. Dario Regis, Andrea Sandri, Ingrid Bonetti, Oscar Bortolami, Pietro Bartolozzi (2012) A Minimum of 10-year Follow-Up of the BurchSchneider Cage and Bulk Allografts for the Revision of Pelvic Discontinuity. The Journal of Arthroplasty, 27(6): 1057-1063.

11. James B Stiehl, Rajit Saluja, Therese Diener (2000) Reconstruction of Major Column Defects and Pelvic Discontinuity in Revision Total Hip Arthroplasty. The Journal of Arthroplasty 15(7): 849-857.

12. Panagiotis P Symeonides, George E Pestatodes, John D Pournaras, George A Kapenatos, Anastatsios G Christodoulou, et al. (2009) The Effectiveness of the Burch-Schneider Antiprotrusio Cage for Acetabular Bone Deficiency. The Journal of Arthrophlasty 24(2): 168-174. 


\section{ISSN: 2574-1241}

DOI: 10.26717/BJSTR.2019.15.002766

S Magersky. Biomed J Sci \& Tech Res

(C) (i) This work is licensed under Creative

Submission Link: https://biomedres.us/submit-manuscript.php

$\begin{array}{ll}\text { BIOMEDICAL } & \text { Assets of Publishing with us } \\ \text { RESEARCHES } & \text { - Global archiving of articles } \\ & \text { - Immediate, unrestricted online access } \\ & \text { - Rigorous Peer Review Process } \\ \end{array}$

